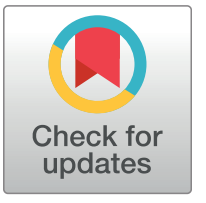

\section{G openaccess}

Citation: Ha J, Jung CW, Choi S, Kim M-G, Gwon JG, Kim JK, et al. (2021) Impact of acute kidney injury on graft outcomes of deceased donor kidney transplantation: A nationwide registry-based matched cohort study in Korea. PLoS ONE 16(11): e0260076. https://doi.org/10.1371/journal. pone.0260076

Editor: Giuseppe Remuzzi, Istituto Di Ricerche Farmacologiche Mario Negri, ITALY

Received: March 12, 2021

Accepted: November 2, 2021

Published: November 17, 2021

Copyright: $\odot 2021 \mathrm{Ha}$ et al. This is an open access article distributed under the terms of the Creative Commons Attribution License, which permits unrestricted use, distribution, and reproduction in any medium, provided the original author and source are credited.

Data Availability Statement: Data can be accessed through Korean Organ Transplantation Registry (KOTRY) website. To gain access to KOTRY data, an application form, a research proposal, and the applicant's institutional review board approval document should be submitted to and reviewed by the Committee of KOTRY. Contact information is as below. Korean Organ Transplantation Registry Website: http://www. kotry.org Tel: +82-2-36752194 Fax: +82-2-3675-2195 E-mail: kotry@kotry. org.

\title{
Impact of acute kidney injury on graft outcomes of deceased donor kidney transplantation: A nationwide registry-based matched cohort study in Korea
}

\begin{abstract}
Jane $\mathrm{Ha}^{1}$, Cheol Woong Jung ${ }^{2 *}$, Sunkyu $\mathrm{Choi}^{3}$, Myung-Gyu Kim ${ }^{4}$, Jun Gyo Gwon ${ }^{2}$, Joong Kyung Kim ${ }^{5}{ }^{5}$, Chan-Duck Kim ${ }^{6}$, Ji Won Min ${ }^{7}$, Jaeseok Yang ${ }^{8}$, Curie Ahn ${ }^{8}$, on behalf of the Korean Organ Transplantation Registry Study group ${ }^{\pi}$

1 Department of Medicine, Korea University College of Medicine, Seoul, Korea, 2 Department of Surgery, Korea University Anam Hospital, Seoul, Korea, 3 Department of Biostatistics, Korea University College of Medicine, Seoul, Korea, 4 Department of Internal Medicine, Korea University Anam Hospital, Seoul, Korea, 5 Department of Internal Medicine, Bongseng Memorial Hospital, Busan, Korea, 6 Department of Internal Medicine, School of Medicine, Kyungpook National University Hospital, Daegu, Korea, 7 Division of Nephrology, Department of Internal Medicine, Bucheon St. Mary's Hospital, Bucheon, Korea, 8 Department of Nephrology, Seoul National University Hospital, Seoul, Korea
\end{abstract}

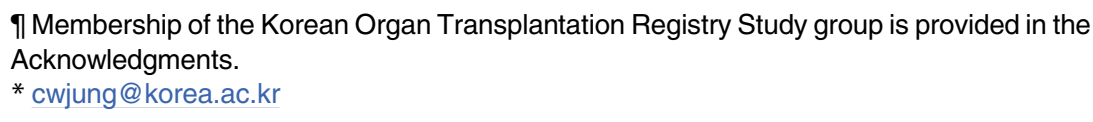

\section{Abstract}

\section{Background}

Favorable long-term and short-term graft survival and patient survival after kidney transplantation (KT) from deceased donors with acute kidney injury (AKI) have been reported. However, few studies have evaluated effects of donor AKI status on graft outcomes after KT in Asian population. Thus, the purpose of this study was to evaluate graft function after KTs from donors with $\mathrm{AKI}$ compared to matched $\mathrm{KT}$ s from donors without AKI using a multicenter cohort in Korea.

\section{Methods}

We analyzed a total of 1,466 KTs collected in Korean Organ Transplant Registry between April 2014 and December 2017. KTs from AKI donors (defined as donors with serum creatinine level $\geq 2 \mathrm{mg} / \mathrm{dL}$ ) and non-AKI donors (275 cases for each group) were enrolled using a 1:1 propensity score matching. Graft outcomes including graft and patient survival, delayed graft function (DGF), rejection rate, and serially measured estimated glomerular filtration rate (eGFR) were evaluated.

\section{Results}

After propensity matching, KTs from AKI donors showed higher rate of DGF ( $44.7 \%$ vs. $24.0 \%, p<0.001)$. However, the rejection rate was not significantly different between the two groups (KTs from AKI donors vs. KTs from non-AKI donors). eGFRs measured after 6 
Funding: This research was supported by research grants (2014-ER6301-00, 2014-ER6301-01, 2014ER6301-02, 2017-ER6301-00, 2017-ER6301-01, 2017-ER6301-02) funded by Korea Centers for Disease Control and Prevention and a Korea University Grant (for C.W.J.). The funders had no role in study design, data collection and analysis, decision to publish, or preparation of the manuscript.

Competing interests: The authors have declared that no competing interests exist. months, 1 year, 2 years and 3 years were not significantly different by donor AKI status. With median follow-up duration of 3.52 years, cox proportional hazards models revealed hazard ratio of 0.973 ( $95 \%$ confidence interval [Cl], 0.584 to 1.621$), 1.004(95 \% \mathrm{Cl}, 0.491$ to $2.054)$ and 0.808 (95\% confidence interval [Cl], 0.426 to 1.532$)$ for overall graft failure, death-censored graft failure and patient mortality, respectively, in KTs from AKI donors compared to KTs from non-AKI donors as a reference.

\section{Conclusions}

KTs from AKI donors showed comparable outcomes to KTs from non-AKI donors, despite a higher incidence of DGF. Results of this study supports the validity of using kidneys from deceased AKI donors in Asian population.

\section{Introduction}

Kidney transplantation (KT) is the treatment of choice for eligible patients with end-stage renal disease (ESRD) which is superior to any other treatment modalities including renal replacement therapies $[1,2]$. However, the issue of organ shortage has been raised because the incidence of ESRD is increasing while the donor pool remains relatively unchanged. There have been efforts to maximize the utilization of donated kidneys. Formalized definition and use of expanded criteria donor (ECD) donor [3] have allowed more patients to benefit from KT.

Kidney discard rate from donors with acute kidney injury (AKI) is significantly higher than that from donors without AKI $[4,5]$ because serum creatinine level of donor has been recognized as one of critical factors contributing to poor outcomes after KT. However, accumulating clinical evidence including long-term observations supports comparable patient survival and graft survival of KT from donors with and without AKI [5-12]. Main concerns about using kidneys from donors with AKI have been primary non function [13]. However, many of previous reports had a single-center design [7, 12, 14-18]. There have not been sufficient studies for supporting the safety and efficacy of KTs from donors with AKI in Asian population [9, 14].

Therefore, we conducted a matched cohort study using a nationwide multicenter cohort in Korea to evaluate effects of deceased donor AKI status on graft function after KT presented by estimated glomerular filtration rate (eGFR) with minimized selection bias, and to assess adequacy of KT from donor with AKI in Asian population.

\section{Patients and methods}

\section{Study population}

The Korean Organ Transplant Registry (KOTRY) is a web-based national transplant registry established in 2014. It includes demographic and clinical data of donors and recipients from 32 centers. The database was accessed on January 2020 for data collection, and all cases of KT from deceased donors and registered in KOTRY between April 2014 and December 2017 were eligible for this study. Exclusion criteria were en-bloc or dual KTs, KTs from donor aged less than 19 years, and cases with missing variables including donor serum creatine, height, weight, presence of hypertension, or presence of diabetes. The KOTRY study was reviewed and approved by the Institutional Review Board of Korea University Anam Hospital (approval number: 2014-0272-024). All data were fully anonymized before researchers accessed the 
database. The Institutional Review Board waived the requirement for informed consent. None of the transplant donors in the dataset was from a vulnerable population and all donors or next of kin provided written informed consent that was freely given. This study followed the Strengthening the Reporting of Observational Studies in Epidemiology reporting guidelines.

\section{Data collection}

Primary data included information of donors, information of recipients, and transplantationrelated factors. Donor factors included age, height, weight, body mass index (BMI), cause of death, serum creatinine, presence of diabetes or hypertension, smoking status, and human leukocyte antigen (HLA) types (HLA-A, -B, -DRB1). Kidney donor profile index (KDPI) and kidney donor risk index (KDRI) were calculated based on donor characteristics [19]. Recipient factors included primary cause of renal disease (diabetes, hypertension, glomerular disease, tubular interstitial disease, polycystic kidney disease, or others), history of kidney transplantation, presence of diabetes or hypertension, age, height, weight, BMI, and HLA types. Transplant-related factors included cold ischemia time (CIT), results of donor specific antigen (DSA) test, agents of induction therapy, and maintenance immunosuppressants. HLA mismatch score was calculated by counting the number of HLA matched loci of HLA-A, -B, and -DRB1 out of six. Clinical outcomes included serum creatinine levels of recipients measured at 6 months, 1 year, 2 years, and 3 years after KT, delayed graft function (DGF, defined as the need for renal replacement therapy during the first week after KT), primary nonfunction (PNF), graft loss, patient loss, biopsy proven rejections within 1-year postKT (borderline rejections, T-cell mediated, antibody mediated, and mixed rejections), and BK nephropathy. eGFR in each follow-up period was calculated using demographic factors and serum creatinine level using the Modification of Diet in Renal Disease study equation [20].

\section{Statistical analysis}

Continuous variables and categorical variables are presented as means with standard deviations and frequency with percentages, respectively. AKI donor was defined as donor with terminal serum creatinine level (the last serum creatinine level measured before KT) $\geq 2.0$ $\mathrm{mg} / \mathrm{dL}$. We used a 1 to 1 propensity score matching from a logistic regression to minimize the difference in baseline covariates between AKI donors and non-AKI donors. We performed propensity score matching in consideration of donor age, sex height, weight, presence of hypertension and diabetes, and cause of death using the caliper matching. To assess the balance of variables used in the matching, standardized mean differences (SMDs) were checked.

Differences in continuous outcomes and categorical outcomes between the two matched groups were investigated using independent t-test and chi-squared test, respectively. A generalized estimating equation model was used to estimate statistical significance of variance in eGFR between groups. Bonferroni correction was used for comparing eGFR at specific time point. Graft failure was defined as a return to dialysis or retransplantation. Kaplan-Meier logrank tests were used to test differences in patient survival, graft survival and death-censored graft failure between groups. The hazard ratios (HRs) and 95\% confidence intervals (CIs) of overall graft failure, death-censored graft failure and death after KT were estimated using Cox proportional hazards model. A $p$-value less than 0.05 in a two-sided test was regarded as statistically significant. All statistical analyses were performed using SAS version 9.4 (SAS Institute, Cary, NC, USA) and R 3.6.3 (Vienna, Austria, http://www.R-project.org). 


\section{Results}

\section{Baseline characteristics of included KTs}

Among 1,466 deceased donor KTs performed between April 2014 and December 2017 and identified from the KOTRY database, $275 \mathrm{KT}$ s from donors with AKI were matched at 1:1 to 275 from donors without AKI according to the propensity score. Fig 1 provides information of excluded cases in detail. Characteristics of matched variables including SMDs by donor AKI status before and after matching are presented in S1 Table.

Baseline characteristics of donors and recipients included in the analysis and transplantation-related characteristics are described in Table 1 according to group. The mean age of donors was $46.7 \pm 13.4$ years. The majority $(83.6 \%)$ of donors were males. Their mean BMI was $23.9 \pm 3.5 \mathrm{~kg} / \mathrm{m}^{2}$. Of all donors, $42.6 \%$ died from cerebrovascular causes. Recipients were $51.1 \pm 10.7$ years old in average. Most $(60.9 \%)$ of them were males. Their mean BMI was $23.1 \pm 3.4 \mathrm{~kg} / \mathrm{m}^{2}$. There were three leading primary causes of renal disease: glomerular disease (35.4\%), diabetes (30.7\%), and hypertension (28.6\%). Mean serum creatinine levels of AKI donors and non-AKI donors were $3.55 \pm 1.44 \mathrm{mg} / \mathrm{dL}$ and $1.05 \pm 0.44 \mathrm{mg} / \mathrm{dL}(p<0.001)$,

\section{1,466 cases of deceased donor KT} performed between April 2014 and December 2017 registered in the KOTRY

\section{$374 \mathrm{KT}$ s were excluded}

167 En-bloc, dual KT, or no information

$48 \mathrm{KTs}$ from donor aged $<19$

26 KTs without presented donor serum creatinine

$134 \mathrm{KTs}$ with missing variables including donor height, weight, presence of hypertension, or presence of diabetes

\section{KTs from donor with AKI and 911 KTs from donor without AKI}

\section{KTs from donor with AKI and 275 KTs from donor without AKI matched 1:1 according to the propensity score}

Fig 1. Flow diagram showing the selection of the study population. 
Table 1. Baseline characteristics of subjects in KT from AKI donor group and KT from non-AKI donor groups.

\begin{tabular}{|c|c|c|c|}
\hline & KT from AKI donor $(n=275)$ & KT from non-AKI donor $(n=275)$ & $P$-value \\
\hline \multicolumn{4}{|l|}{ Donor characteristics } \\
\hline Age, years & $47.17 \pm 12.11$ & $46.29 \pm 14.58$ & 0.443 \\
\hline Male, No. (\%) & $227(82.5)$ & $233(84.7)$ & 0.564 \\
\hline Height, $\mathrm{cm}$ & $169.48 \pm 8.86$ & $170.20 \pm 8.11$ & 0.323 \\
\hline Weight, kg & $69.40 \pm 11.75$ & $68.83 \pm 12.35$ & 0.577 \\
\hline $\mathrm{BMI}, \mathrm{kg} / \mathrm{m}^{2}$ & $24.10 \pm 3.48$ & $23.66 \pm 3.52$ & 0.147 \\
\hline Diabetes, No. (\%) & $45(16.4)$ & $44(16.0)$ & 1.000 \\
\hline Hypertension, No. (\%) & $79(28.7)$ & $73(26.5)$ & 0.634 \\
\hline Serum creatinine, $\mathrm{mg} / \mathrm{dL}$ & $3.55 \pm 1.44$ & $1.05 \pm 0.44$ & $<0.001$ \\
\hline Cerebrovascular death, No. (\%) & $119(43.3)$ & $115(41.8)$ & 0.796 \\
\hline Current or ex-smoker, No. (\%) & $145(55.6)$ & $127(50.2)$ & 0.259 \\
\hline ECMO, No. (\%) & $14(5.1)$ & $9(3.3)$ & 0.394 \\
\hline KDRI & $1.66 \pm 0.40$ & $1.42 \pm 0.38$ & $<0.001$ \\
\hline KDPI, \% & $75 \pm 18$ & $61 \pm 22$ & $<0.001$ \\
\hline \multicolumn{4}{|l|}{ Recipient characteristics } \\
\hline Age, years & $51.83 \pm 10.45$ & $50.36 \pm 10.72$ & 0.106 \\
\hline Male, No. (\%) & $172(62.5)$ & $163(59.3)$ & 0.484 \\
\hline Height, $\mathrm{cm}$ & $165.79 \pm 8.25$ & $164.65 \pm 8.65$ & 0.114 \\
\hline Weight, kg & $63.12 \pm 11.16$ & $63.21 \pm 12.23$ & 0.935 \\
\hline $\mathrm{BMI}, \mathrm{kg} / \mathrm{m}^{2}$ & $22.88 \pm 3.14$ & $23.22 \pm 3.57$ & 0.235 \\
\hline Diabetes, No. (\%) & $82(29.7)$ & $80(29.1)$ & 0.925 \\
\hline Hypertension, No. (\%) & $254(92.7)$ & $243(88.4)$ & 0.112 \\
\hline Current or ex-smoker, No. (\%) & $78(28.6)$ & $59(21.5)$ & 0.072 \\
\hline Past history of KT, No. (\%) & $22(8.0)$ & $20(7.27)$ & 0.748 \\
\hline Duration of dialysis before KT, years & $7.45 \pm 4.90$ & $7.54 \pm 4.74$ & 0.832 \\
\hline \multicolumn{4}{|l|}{ Primary cause of renal disease, No. (\%) } \\
\hline Diabetes & $71(32.0)$ & $62(29.4)$ & \multirow[t]{6}{*}{ P for trend 0.244} \\
\hline Hypertension & $69(31.1)$ & $55(26.1)$ & \\
\hline Glomerular disease & $70(31.5)$ & $83(39.3)$ & \\
\hline Tubulointerstitial disease & $0(0.0)$ & $2(0.9)$ & \\
\hline Polycystic kidney disease & $12(5.4)$ & $9(4.3)$ & \\
\hline Other or unknown & $53(19.3)$ & $64(23.3)$ & \\
\hline \multicolumn{4}{|l|}{ Transplantation characteristics } \\
\hline CIT, hours & $4.90 \pm 2.22$ & $4.97 \pm 2.09$ & 0.748 \\
\hline HLA mismatch score, out of 6 & $2.29 \pm 1.63$ & $2.47 \pm 1.71$ & 0.202 \\
\hline Baseline DSA positive, No. (\%) & $18(11.3)$ & $26(15.3)$ & 0.370 \\
\hline \multicolumn{4}{|l|}{ Induction medication, No. (\%) } \\
\hline Anti-thymocyte globulin & $123(44.7)$ & $66(24.0)$ & $<0.001$ \\
\hline Basiliximab & $152(55.3)$ & $209(76.0)$ & $<0.001$ \\
\hline \multicolumn{4}{|c|}{ Maintenance immunosuppressants, No. (\%) } \\
\hline Tacrolimus & $270(98.2)$ & $273(99.3)$ & 0.450 \\
\hline Cyclosporin & $4(1.5)$ & $0(0)$ & 0.124 \\
\hline Mycophenolic acid & $262(95.3)$ & $258(93.8)$ & 0.453 \\
\hline Steroid & $272(98.9)$ & $268(97.5)$ & 0.202 \\
\hline
\end{tabular}

KT, kidney transplantation; AKI, acute kidney injury; SMD, standardized mean difference; BMI, body mass index; ECMO, extracorporeal membrane oxygenation; KDPI, kidney donor profile index; KDRI, kidney donor risk index; CIT, cold ischemic time; HLA, human leukocyte antigen; DSA, donor-specific antibody.

https://doi.org/10.1371/journal.pone.0260076.t001 
Table 2. Graft outcomes by AKI status of deceased donor.

\begin{tabular}{l|l|l|l}
\hline Variables & KT from AKI donor & KT from non-AKI donor & P-value \\
\hline eGFR, $\mathrm{mL} / \mathrm{min} / 1.73 \mathrm{~m}^{2}$ & & & \\
\hline 6 months post-KT & $53.29 \pm 20.60$ & $55.94 \pm 23.44$ & 0.172 \\
\hline 1 year post-KT & $55.20 \pm 19.93$ & $57.28 \pm 22.69$ & 0.279 \\
\hline 2 years post-KT & $56.53 \pm 20.20$ & $60.56 \pm 22.80$ & 0.064 \\
\hline 3 years post-KT & $55.71 \pm 19.32$ & $60.25 \pm 22.19$ & 0.099 \\
\hline PNF, No. (\%) & $2(0.73)$ & $0(0.00)$ & 0.157 \\
\hline DGF, No. $(\%)$ & $67(24.54)$ & $17(6.18)$ & $<0.001$ \\
\hline Biopsy-confirmed rejection within 1 year, No. (\%) & $43(15.64)$ & $54(19.64)$ & 0.2184 \\
\hline Acute T cell-mediated rejection & $17(9.71)$ & $20(7.27)$ & 0.7031 \\
\hline Acute antibody-mediated rejection & $16(5.82)$ & $24(8.73)$ & $7(2.55)$ \\
\hline Mixed acute rejection & $5(1.82)$ & $3(1.09)$ & \\
\hline Biopsy-confirmed BK nephropathy, No. $(\%)$ & $5(1.82)$ & $6(2.18)$ & 0.504 \\
\hline
\end{tabular}

KT, kidney transplantation; AKI, acute kidney injury; eGFR, estimated glomerular filtration rate; DGF, delayed graft function; PNF, primary nonfunction

respectively. Compared to non-AKI donors, AKI donors had higher mean KDRI ( $1.66 \pm 0.40$ versus $1.42 \pm 0.38, p<0.001)$ and KDPI $(75 \pm 18 \%$ versus $61 \pm 22 \%, p<0.001)$. Anti-thymocyte globulin was more frequently used as an induction agent in KTs from AKI donors than in KTs from non-AKI donors ( $44.7 \%$ versus $24.0 \%, p<0.001$ ). No significant difference in the frequency of patients using each maintenance immunosuppressant was observed.

\section{Allograft outcomes}

Allograft outcomes according to donor AKI status are presented in Table 2. The incidence of DGF was significantly higher in the group of KTs from AKI donors than in the group of KTs from non-AKI donors $(24.5 \%$ versus $6.2 \%, p<0.001)$. However, the incidence of PNF was not significantly different between the two groups $(p=0.157)$. There was a significant difference in eGFR by time ( $p=0.006$, Fig 2 ), although the variance in eGFR was not significantly different between KTs from AKI and non-AKI donors ( $p=0.427)$. After KTs from non-AKI donors, eGFR was improved at 3 years compared to that at 6 months after KT $(p=0.002)$. There was no significant difference in the incidence of biopsy-confirmed rejection within 1 year or biopsy-confirmed BK nephropathy.

\section{Allograft and patient survival}

The median follow-up duration of recipients was 3.52 (interquartile range, 2.69-4.28) years. Graft and patient survivals by donor AKI status are shown in Fig 3. The risk of overall graft failure (HR, 0.973; 95\% CI, 0.584 to 1.621), death-censored graft failure (HR, 1.004; 95\% CI, 0.491 to 2.054 ) and mortality (HR, 0.808 ; $95 \%$ CI, 0.426 to 1.532 ) were not significantly different by donor AKI status.

\section{Discussion}

The discard rate of deceased kidneys is increasing without showing significant difference in the quality between transplanted and discarded kidneys [21], implying that better assessment and distribution of such limited resource could increase the donor pool. Given that AKI donor is one of common causes of kidney discard, the impact of donor AKI status on outcomes of 




Fig 2. Mean eGFR in KT from AKI donors and non-AKI donors. eGFR, estimated glomerular filtration rate; KT, kidney transplantation; AKI, acute kidney injury. Generalized estimating equation showed significant difference in eGFR by time $(p=0.006)$. However, group versus time interaction was insignificant $(p=0.427)$. eGFR showed a significant difference $\left({ }^{*}, p<0.05\right)$ at 6 months and 3 years after KT.

KT comes to the fore. Although preceding studies have reported favorable outcomes of KTs from AKI donors $[4-8,10,12-14,17,18]$ and feasible mechanisms have been proposed [11, 22], there have been considerable discordances in the selection criteria for kidneys from AKI

A

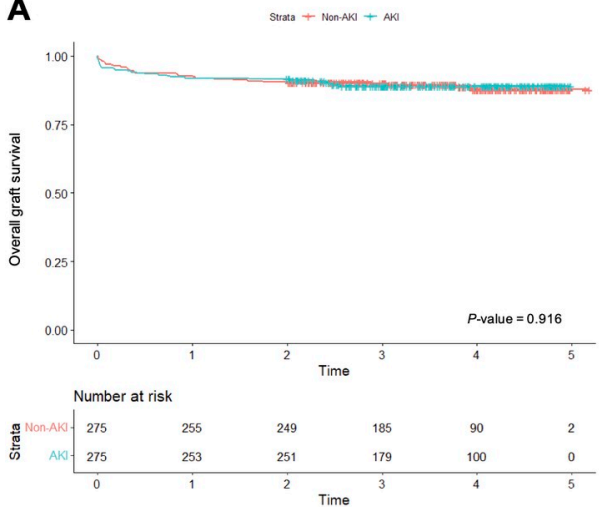

B

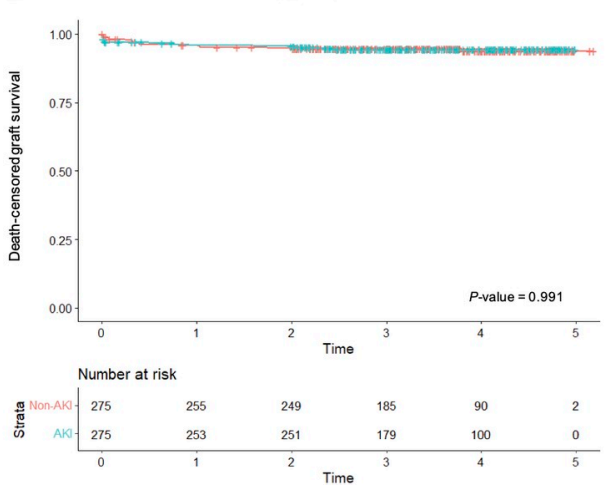

C



Fig 3. Overall graft survival (A), death-censored graft survival (B) and patient survival (C) after KT from donor with AKI and without AKI. 
donors across centers [7, 12]. Due to low feasibility of randomized controlled trials, welldesigned observational studies with low risk of bias and confounders could provide decisive evidence.

To the best of our knowledge, this is the first nationwide cohort study reporting serial graft function after KTs according to donor AKI status in an Asian population. We found that KTs from AKI donors resulted in comparable outcomes to propensity score-matched KTs from non-AKI donors in an Asian population. According to our findings, despite higher incidence of DGF in KTs from AKI donors, the rejection rate, eGFR, graft survival, and patient survival were not significantly affected by donor AKI status. These findings are in line with prior studies including one study on 6,722 deceased donors with AKI matched to donors without AKI showing that AKI status of donor can significantly increase the risk of DGF ( $29 \%$ versus $22 \%$, $\mathrm{p}<0.001$ ), while AKI status was not related to death-censored graft failure (HR: 1.01; 95\% CI: 0.95 to 1.08 ) or all-cause graft failure (HR: $0.97 ; 95 \%$ CI: 0.93 to 1.02) [11]. Higher incidence of DGF relevant to donor AKI has been consistently featured in abundant studies. However, convincing short-term and long-term outcomes justify the use of AKI kidneys [23].

Our study population composed of Asian with short CIT (mean, $4.94 \pm 2.16$ hours), one of the most important factors affecting graft survival and function [24, 25]. In addition, basiliximab was predominantly prescribed as an induction agent attributing to a low immunologic risk of Asian [26]. Before cases were matched by propensity score, the study cohort showed that AKI donors tended to be taller and heavier than non-AKI donors (S1 Table). This tendency can be explained by choosing favorable characteristics offsetting higher risk of poor prognosis after KT. The size of kidney, which is proportional to the body size of the donor, is related to better prognosis after KT [27, 28].

We observed a significant increase in kidney function measured at 3 years compared to 6 months after KTs from non-AKI donors (Fig 2), which was also reported in recent studies [29, 30]. Although the exact mechanism has not been elucidated yet and its clinical significance is controversial, several plausible explanations could be suggested. First, improved kidney function over time could be explained as a compensatory hyperfiltration of the graft in a fashion similar to improvement of renal function in live donors after nephrectomy [31]. Second, improvements in general medical care for transplant patients and management for high immunologic risk patients and acute rejection episodes with better choice of immune suppression $[32,33]$ might have contributed to better graft functions after KT. Third, increase in eGFR within 3 years after KTs could be explained as a natural course of recovery from pre-, intra-, and post-transplant graft injury such as ischemic insults during the donor/recipient management and ischemia reperfusion injury which is an inevitable consequence following KTs [33].

In this study, there was no significant difference in the mean eGFR measured at each follow-up period between KTs from AKI and KTs from non-AKI donors. Previous long-term observation studies with multiple eGFR measurements to compare graft functions between KTs from AKI and non-AKI donors have shown inconsistent results. Some studies showed that eGFR after KTs from AKI donors was comparable to that after KTs from non-AKI donors until 5 years $[12,17]$. In contrast, Bauer et al. [18] found that KTs from AKI and non-AKI donors did not show significant difference in eGFR measured at 1 year after KT; however, eGFR at 3 and 5 years after KT was significantly higher in the non-AKI group than in the AKI group. Schütte-Nütgen et al. [34] also reported that KTs from AKI donors consistently show lower eGFR than KTs from non-AKI donors at 3-year follow-up.

Some limitations of this study should be acknowledged. First, we used dichotomized definition of AKI donors without considering chronicity, severity, or dynamic changes in donor kidney function as donor serum creatinine level was reported only once in the registry. Second, 
although bias from the retrospective design of this study was partially resolved by propensity score matching, there might be effects of known and unknown variables. Third, a considerable number of cases were excluded due to missing variables. Fourth, the small cohort size decreased the statistical power of this study.

Taken together, this study supports the validity of using kidneys from deceased AKI donors in Asian population. Further studies with long term follow-up duration are needed to investigate graft function according to donor AKI status.

\section{Supporting information}

S1 Table. Characteristics of matched variables according to donor AKI status before and after propensity score matching. (DOCX)

\section{Acknowledgments}

The membership of the Korean Organ Transplantation Registry Study group is as follows. The leader of this group is Curie Ahn (curie@snu. ac.kr).

Curie $\mathrm{Ahn}^{1}$, Jaeseok Yang ${ }^{2}$, Jin Min Kong ${ }^{3}$, Oh Jung Kwon ${ }^{4}$, Deok Gie Kim ${ }^{5}$, Cheol Woong Jung ${ }^{6}$, Yeong Hoon Kim ${ }^{7}$, Joong Kyung Kim ${ }^{8}$, Chan-Duck Kim ${ }^{9}$, Ji Won Min ${ }^{10}$, Sung Kwang Park $^{11}$, Yeon Ho Park ${ }^{12}$, Park Jae Berm ${ }^{13}$, Jung Hwan Park ${ }^{14}$, Jong-Won Park ${ }^{15}$, Tae Hyun Ban $^{16}$, Sang Heon Song ${ }^{17}$, Seung Hwan Song ${ }^{18}$, Ho Sik Shin ${ }^{19}$, Chul Woo Yang ${ }^{20}$, Hye Eun Yoon $^{21}$, Kang Wook Lee ${ }^{22}$, Dong Ryeol Lee ${ }^{23}$, Dong Won Lee ${ }^{24}$, Sam Yeol Lee ${ }^{25}$, Sang-Ho $\mathrm{Lee}^{26}$, Su Hyung Lee ${ }^{27}$, Jung Jun Lee ${ }^{28}$, Lee Jung $\mathrm{Pyo}^{29}$, Jeong-Hoon Lee ${ }^{30}$, Jin Seok Jeon ${ }^{31}$, Heungman Jun ${ }^{32}$, Kyunghwan Jeong ${ }^{33}$, Ku Yong Chung ${ }^{34}$, Hong Rae Cho ${ }^{35}$, Ju Man Ki ${ }^{36}$, Dong-Wan Chae ${ }^{37}$, Soo Jin Na Choi ${ }^{38}$, Duck Jong $\mathrm{Han}^{39}$, Seungyeup $\mathrm{Han}^{40}$, Kyu Ha $\mathrm{Huh}^{41}$

${ }^{1}$ Department of Nephrology, Seoul National University Hospital

${ }^{2}$ Department of Surgery, Seoul National University Hospital

${ }^{3}$ Department of Nephrology, BHS Hanseo Hospital

${ }^{4}$ Department of Surgery, College of Medicine, Han Yang University

${ }^{5}$ Department of Surgery, Yonsei University Wonju College of Medicine, Wonju Severance Christian Hospital

${ }^{6}$ Department of Transplantation and Vascular Surgery, Korea University Anam Hospital

${ }^{7}$ Department of Internal Medicine, Inje University Busan Paik Hospital

${ }^{8}$ Department of Internal Medicine, Bongseng Memorial Hospital

${ }^{9}$ Department of Internal Medicine, School of Medicine, Kyungpook National University Hospital

${ }^{10}$ Division of Nephrology, Department of Internal Medicine, Bucheon St. Mary's Hospital

${ }^{11}$ Department of Internal Medicine, Chonbuk National University Medical School

${ }^{12}$ Department of Surgery, Gil Medical Center, Gachon University College of Medicine

${ }^{13}$ Department of Surgery, Samsung Medical Center, Sungkyunkwan University School of Medicine

${ }^{14}$ Konkuk University School of Medicine, Department of Nephrology

${ }^{15}$ Department of Nephrology, Yeungnam University Hospital

${ }^{16}$ Division of Nephrology, Department of Internal Medicine, Eunpyeong St. Mary's hospital

${ }^{17}$ Organ Transplantation Center and Department of Internal Medicine, Pusan National University Hospital

${ }^{18}$ Department of Surgery, Ewha Womans University Medical Center 
${ }^{19}$ Kosin University College of Medicine, Department of Internal Medicine, Division of Nephrology

${ }^{20}$ Division of Nephrology, Department of Internal Medicine, Seoul St. Mary's hospital

${ }^{21}$ Department of Internal Medicine, Incheon St. Mary's Hospital

${ }^{22}$ Department of Nephrology, Chungnam National University Hospital

${ }^{23}$ Division of Nephrology, Department of Internal Medicine, Maryknoll Medical Center

${ }^{24}$ Division of Nephrology, Department of Internal Medicine, Pusan National University School of Medicine

${ }^{25}$ Department of Surgery, Kangdong Sacred Heart Hospital, Hallym University College of Medicine

${ }^{26}$ Department of Nephrology, Kyung Hee University Hospital at Gangdong

${ }^{27}$ Department of Surgery, Ajou University School of Medicine

${ }^{28}$ Department of Surgery, CHA Bundang Medical Center

29 Department of Nephrology, SNU Boramae Medical Center

${ }^{30}$ Department of Surgery, Myongji Hospital

${ }^{31}$ Department of Internal Medicine, Soonchunhyang University Seoul Hospital

32 Department of Surgery, Inje University Ilsan Paik Hospital

${ }^{33}$ Department of Internal Medicine, Kyung Hee University College of Medicine

${ }^{34}$ Department of Surgery, Ewha Womans University Mokdong Hospital

35 Department of Surgery, Ulsan University Hospital

${ }^{36}$ Department of Surgery, Gangnam Severance Hospital, Yonsei University College of Medicine

${ }^{37}$ Division of Nephrology, Seoul National University Bundang Hospital

${ }^{38}$ Department of Surgery, Chonnam National University Medical School

${ }^{39}$ Department of Surgery, Asan Medical Center

${ }^{40}$ Department of Internal Medicine, Keimyung University School of Medicine

${ }^{41}$ Department of Transplantation Surgery, Severance Hospital

\section{Author Contributions}

Conceptualization: Cheol Woong Jung, Joong Kyung Kim, Curie Ahn.

Data curation: Sunkyu Choi, Joong Kyung Kim.

Formal analysis: Sunkyu Choi, Jun Gyo Gwon, Jaeseok Yang.

Investigation: Jane Ha, Cheol Woong Jung, Myung-Gyu Kim, Chan-Duck Kim, Ji Won Min.

Supervision: Cheol Woong Jung.

Validation: Jun Gyo Gwon.

Writing - original draft: Jane Ha, Cheol Woong Jung, Jaeseok Yang.

Writing - review \& editing: Myung-Gyu Kim, Jun Gyo Gwon, Chan-Duck Kim, Ji Won Min, Curie Ahn.

\section{References}

1. Purnell TS, Auguste $P$, Crews DC, et al. Comparison of life participation activities among adults treated by hemodialysis, peritoneal dialysis, and kidney transplantation: a systematic review. American Journal of Kidney Diseases. 2013; 62:953-973. https://doi.org/10.1053/j.ajkd.2013.03.022 PMID: 23725972

2. Schold JD, Buccini LD, Goldfarb DA, Flechner SM, Poggio ED, Sehgal AR. Association between kidney transplant center performance and the survival benefit of transplantation versus dialysis. Clinical 
Journal of the American Society of Nephrology. 2014; 9:1773-1780. https://doi.org/10.2215/CJN. 02380314 PMID: 25237071

3. Port FK, Bragg-Gresham JL, Metzger RA, et al. Donor characteristics associated with reduced graft survival: an approach to expanding the pool of kidney donors. Transplantation. 2002; 74:1281-1286. https://doi.org/10.1097/00007890-200211150-00014 PMID: 12451266

4. Hall IE, Schröppel B, Doshi MD, et al. Associations of deceased donor kidney injury with kidney discard and function after transplantation. American Journal of Transplantation. 2015; 15:1623-1631. https:// doi.org/10.1111/ajt.13144 PMID: 25762442

5. Kayler L, Garzon P, Magliocca J, et al. Outcomes and utilization of kidneys from deceased donors with acute kidney injury. American Journal of Transplantation. 2009; 9:367-373. SRTR https://doi.org/10. 1111/j.1600-6143.2008.02505.x PMID: 19178415

6. Hall IE, Akalin E, Bromberg JS, et al. Deceased-donor acute kidney injury is not associated with kidney allograft failure. Kidney international. 2019; 95:199-209. multicenter https://doi.org/10.1016/j.kint.2018. 08.047 PMID: 30470437

7. Heilman RL, Smith ML, Smith BH, et al. Long-term outcomes following kidney transplantation from donors with acute kidney injury. Transplantation. 2019; 103:e263-e272. Single center https://doi.org/ 10.1097/TP.0000000000002792 PMID: 31205261

8. Kumar MSA, Khan SM, Jaglan S, et al. Successful transplantation of kidneys from deceased donors with acute renal failure: three-year results. Transplantation. 2006; 82:1640-1645. https://doi.org/10 1097/01.tp.0000250908.62948.8f PMID: 17198251

9. Kim JH, Kim YS, Choi MS, et al. Prediction of clinical outcomes after kidney transplantation from deceased donors with acute kidney injury: a comparison of the KDIGO and AKIN criteria. BMC nephrology. 2017; 18:39. 강남, 의정부 성모 https://doi.org/10.1186/s12882-017-0461-5 PMID: 28129763

10. Klein R, Galante NZ, de Sandes-Freitas TV, de Franco MF, Tedesco-Silva H, Medina-Pestana JO. Transplantation with kidneys retrieved from deceased donors with acute renal failure. Transplantation. 2013; 95:611-616. https://doi.org/10.1097/TP.0b013e318279153c PMID: 23274968

11. Liu C, Hall IE, Mansour S, Philbrook HRT, Jia Y, Parikh CR. Association of deceased donor acute kidney injury with recipient graft survival. JAMA Network Open. 2020; 3:e1918634. https://doi.org/10.1001/ jamanetworkopen.2019.18634 PMID: 31913491

12. Jadloweic $C C$, Heilman $R L$, Smith $M L$, et al. Transplanting kidneys from donation after cardiac death donors with acute kidney injury. American Journal of Transpalntation. 2020; 20:864-869. Single center

13. Boffa $C$, Van de Leemkolk F, Curnow E, et al. Transplantation of kidneys from donors with acute kidney injury: friend or foe? American Journal of Transplantation. 2017; 17:411-419. https://doi.org/10.1111/ ajt.13966 PMID: 27428556

14. Jung CW, Park K, Kim S, et al. Clinical outcomes in kidney transplantation patients from deceased donors with acute kidney injury. Transplantation proceedings. Elsevier; 2013: 2941-2945. Single center, short term https://doi.org/10.1016/j.transproceed.2013.08.048 PMID: 24157008

15. Heilman R, Smith $\mathrm{M}$, Kurian $\mathrm{S}$, et al. Transplanting kidneys from deceased donors with severe acute kidney injury. American Journal of Transplantation. 2015; 15:2143-2151. Single center https://doi.org/ 10.1111/ajt.13260 PMID: 25808278

16. Farney AC, Rogers J, Orlando G, et al. Evolving experience using kidneys from deceased donors with terminal acute kidney injury. Journal of the American College of Surgeons. 2013;216:645-655. https:// doi.org/10.1016/j.jamcollsurg.2012.12.020 PMID: 23395159

17. Domagala $\mathrm{P}$, Gorski L, Wszola M, et al. Successful transplantation of kidneys from deceased donors with terminal acute kidney injury. Renal failure. 2019; 41:167-174. https://doi.org/10.1080/0886022X. 2019.1590209 PMID: 30909784

18. Bauer J, Grzella S, Bialobrzecka M, et al. Success of kidney transplantations from deceased donors with acute kidney injury. Annals of transplantation. 2018; 23:836. https://doi.org/10.12659/AOT.912660 PMID: 30523243

19. Rao PS, Schaubel DE, Guidinger MK, et al. A comprehensive risk quantification score for deceased donor kidneys: the kidney donor risk index. Transplantation. 2009; 88:231-236. https://doi.org/10.1097/ TP.0b013e3181ac620b PMID: 19623019

20. Stevens LA, Coresh J, Greene T, Levey AS. Assessing kidney function-measured and estimated glomerular filtration rate. New England Journal of Medicine. 2006; 354:2473-2483. https://doi.org/10. 1056/NEJMra054415 PMID: 16760447

21. Mohan S, Chiles MC, Patzer RE, et al. Factors leading to the discard of deceased donor kidneys in the United States. Kidney international. 2018; 94:187-198. https://doi.org/10.1016/j.kint.2018.02.016 PMID: 29735310 
22. Puthumana J, Hall IE, Reese PP, et al. YKL-40 associates with renal recovery in deceased donor kidney transplantation. Journal of the American Society of Nephrology. 2017; 28:661-670. https://doi.org/ 10.1681/ASN.2016010091 PMID: 27451287

23. Chan GCK, Chow KM. Should we use kidneys from donors with acute kidney injury for renal transplantation? Nephrology. 2020; 25:105-115. https://doi.org/10.1111/nep.13679 PMID: 31707757

24. Summers DM, Johnson RJ, Allen J, et al. Analysis of factors that affect outcome after transplantation of kidneys donated after cardiac death in the UK: a cohort study. The Lancet. 2010; 376:1303-1311. https://doi.org/10.1016/S0140-6736(10)60827-6 PMID: 20727576

25. Kayler L, Magliocca J, Zendejas I, Srinivas T, Schold J. Impact of cold ischemia time on graft survival among ECD transplant recipients: a paired kidney analysis. American journal of transplantation. 2011; 11:2647-2656. https://doi.org/10.1111/j.1600-6143.2011.03741.x PMID: 21906257

26. Chang J-Y, Yu J, Chung BH, et al. Immunosuppressant prescription pattern and trend in kidney transplantation: A multicenter study in Korea. PloS one. 2017; 12:e0183826. https://doi.org/10.1371/journal. pone.0183826 PMID: 28846737

27. Miles AMV, Sumrani N, John S, et al. The effect of kidney size on cadaveric renal allograft outcome. Transplantation. 1996; 61:894-897. https://doi.org/10.1097/00007890-199603270-00009 PMID: 8623156

28. Moreso F, Serón D, Anunciada Al, et al. Recipient body surface area as a predictor of posttransplant renal allograft evolution. Transplantation. 1998; 65:671-676. https://doi.org/10.1097/00007890199803150-00012 PMID: 9521202

29. Gourishankar S, Hunsicker LG, Jhangri GS, Cockfield SM, Halloran PF. The stability of the glomerular filtration rate after renal transplantation is improving. Journal of the American Society of Nephrology. 2003; 14:2387-2394. https://doi.org/10.1097/01.asn.0000085019.95339.fO PMID: 12937318

30. Srinivas TR, Flechner SM, Poggio ED, et al. Glomerular filtration rate slopes have significantly improved among renal transplants in the United States. Transplantation. 2010; 90:1499-1505. https://doi.org/10. 1097/TP.0b013e3182003dda PMID: 21085061

31. Bertolatus JA, Friedlander MA, Scheidt C, Hunsicker LG. Urinary albumin excretion after donor nephrectomy. American Journal of Kidney Diseases. 1985; 5:165-169. https://doi.org/10.1016/s02726386(85)80045-7 PMID: 3883759

32. Lim MA, Kohli J, Bloom RD. Immunosuppression for transplantation: Where are we now and where are we going? Transplantation Reviews. 2017; 31:10-17. https://doi.org/10.1016/j.trre.2016.10.006 PMID: 28340885

33. Salvadori M, Rosso G, Bertoni E. Update on ischemia-reperfusion injury in kidney transplantation: Pathogenesis and treatment. World Journal of Transplantation. 2015; 5:52-67. https://doi.org/10.5500/wjt. v5.i2.52 PMID: 26131407

34. Schütte-Nütgen K, Finke M, Ehlert S, et al. Expanding the donor pool in kidney transplantation: Should organs with acute kidney injury be accepted?-A retrospective study. PloS one. 2019; 14:e0213608. https://doi.org/10.1371/journal.pone.0213608 PMID: 30865677 